\section{Bigger than 20 Olympic pools}

Tokyo

AFTER years of lobbying by Tokyo University researchers, the Ministry of Education, Science and Culture finally submitted a budget request at the end of last month for a giant neutrino detector to be built in a mine in Gifu Prefecture. SuperKamiokande, which is expected to be completed in 1996, will be the largest and most sensitive observatory for neutrinos and nucleon decay in the world.

The device is a scaled-up version of the Kamiokande detector which made headlines in science in 1987 by detecting neutrinos from the supernova in the Large Magellanic Cloud. The new detector, which will be more than ten times the size of Kamiokande, will contain 50,000 tons of water - enough to fill more than 20 Olympic swimming pools - and it will be able to detect and trace the source of neu-

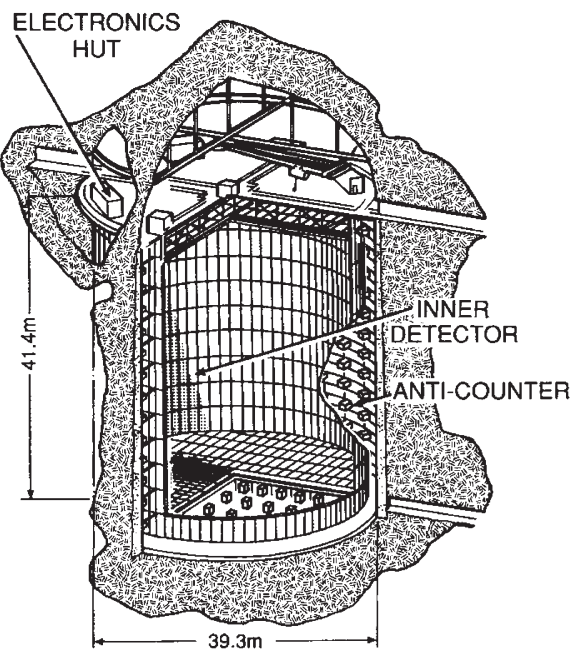

The planned Super-Kamiokande detector trinos from supernovae and even to pinpoint supernovae too faint to be detected by optical telescopes. Furthermore, with fast on-line analysis by computer, SuperKamiokande will be able to locate supernovae and inform optical observatories of their position before the exploding stars become bright, say researchers at the Institute of Cosmic Ray Research of Tokyo University who will build the detector.

Super-Kamiokande should also be able to pick up the relic cosmic particles from formation of the early Universe, such as relic neutrinos from past supernova. And it may even detect the formation of black holes from the abrupt disappearance of neutrino bursts when a neutrino signal is cut-off by black hole formation, the Tokyo researchers say.

The detector works by picking up Cerenkov radiation from high-energy electrons produced, for example, when a neutrino enters the tank or when a proton in the water decays. The inner walls of the tank will be lined with 11,200 highly sensitive photomultiplier tubes to detect and multiplier tubes in the outer wall will help to filter out background radiation (see figure). $1 \mathrm{~km}$ underground to screen out unwanted cosmic radiation. And it will be filled with tion, will be sufficiently transparent for the photomultiplier tubes to pick up tiny bursts of Cerenkov radiation even from the centre of the gigantic tank.

The education ministry has applied for $¥ 613$ million ( $\$ 4.5$ million) in next fiscal locate the radiation. Another 700 photo-

The tank will be encased in solid rock water from the mine, which, after filtra-

\section{Tokyo}

Now that one group of physicists at Tokyo University has won funding for the giant neutrino observatory Super-Kamiokande (see above), a second group is expected to push even harder for its plan to build a huge new accelerator complex equipped with a $1-\mathrm{GeV}$ proton linear accelerator (linac). If approved, the Japan Hadron Project will not only cost hundreds of millions of dollars, it will also involve a major reorganization of Tokyo University and could lead to the formation of a new national research institute to run the facility.

The project has been promoted for several years by the university's Institute for Nuclear Study. Preliminary studies and committee meetings to discuss the project were supported this fiscal year with $¥ 4$ million $(\$ 30,000)$ from the Ministry of Education, Science and Culture. The ministry is expected to provide more funds next year for an in-depth study of the project and the related reorganization of Tokyo University that it would require.

Under the institute's plan, a 1-GeV proton linear accelerator would be built next to the High Energy Physics Laboratory (KEK) in Tsukuba, which houses the huge positron-electron collider TRISTAN and other accelerator facilities. As well as feeding $1-\mathrm{GeV}$ protons to an existing 12 $\mathrm{GeV}$ proton synchrotron in $\mathrm{KEK}$ - an arrangement that would substantially improve the quality and intensity of the KEK synchrotron beam - the proton accelerator would supply beams of protons to a meson science facility and to a neutron scattering facility. The protons would also erator that would produce beams of exotic nuclei.

The great variety of beams available in the proposed complex would make possible a wide range of experiments in condensed-matter physics, chemistry and the life sciences, as well as in particle and be injected into a heavy-ion linear accel- year's budget to cover the costs of the first 2,000 of the huge 20 -inch photomultiplier tubes. Hamamatsu Photonics, a small Japanese company, is the world's only manufacturer of such tubes, which must be meticulously put together by hand.

The education ministry estimates that the overall cost of Super-Kamiokande will be about $¥ 8,700$ million ( $\$ 64$ million). Funds for excavation of the gigantic hole for the detector have already been approved in this year's budget. And Mitsui Mining and Smelting Company, which owns the mine in Gifu Prefecture, will begin preparatory work for the excavation next month.

David Swinbanks

\title{
Is a giant linac next?
}

nuclear physics. And proponents of the scheme are calling for the creation of a new 'national research institute for joint university use' to operate the accelerator complex.

Such national research institutes are among the best-funded organizations run by the education ministry, and they include KEK and the Institute of Space and Astronautical Science. The Institute for Nuclear Study has been lobbying hard to be designated as a national research institute. If it succeeds, it will follow in the footsteps of the National Astronomical Observatory and the Institute of Space and Astronautical Science, both of which were part of Tokyo University.

But there is some resistance to such a move within other parts of the university. Among the contentious questions to be examined next year are: Who will teach nuclear studies at Tokyo University if the institute separates from the university? What effect will the move have on the closely related Institute of Cosmic Ray Research, which branched off from the institute in 1976? Senior university officials want to ensure that the new facility maintains close links with the university, and it is still unclear how the proposed new institute would interact with KEK next door.

The total cost of the new complex is variously estimated at $¥ 30,000$ million to $¥ 40,000$ million ( $\$ 220-300$ million), excluding the cost of the building to house the accelerators. Construction of related facilities attached to the $12-\mathrm{GeV}$ synchrotron at KEK has already begun. A few years ago, Tokyo University decided to give priority to the Super-Kamiokande project in its budget negotiations with the education ministry (Nature 331, 379; 1988), and now that Super-Kamiokande is under way, a push to launch the Japan Hadron Project can be expected. But approval is unlikely for at least a year or two.

David Swinbanks 